\title{
An open bosonic string with one end fixed
}

\author{
Umut Gürsoy $^{a, b}$ and Cihan Saçlıoglu ${ }^{c, b}$ \\ ${ }^{a}$ Center for Theoretical Physics, MIT, 02139 Cambridge, Massachusetts, USA \\ ${ }^{b}$ Feza Gürsey Institute, TUBITAK-Bog̃aziçi University, 81220, Istanbul, Turkey \\ ${ }^{c}$ Physics Department, Bog̃aziçi University, 80815 Bebek, Istanbul, Turkey
}

\begin{abstract}
We study a bosonic string with one end free and the other confined to a D0-brane. Only the odd oscillator modes are allowed, which leads to a Virasoro algebra of even Virasoro modes only. The theory is quantized in a gauge where world-sheet time and ordinary time are identified. There are no negative or null norm states, and no tachyon. The Regge slope is twice that of the open string; this can serve as a test of the usefulness of the the model as a semi-quantitative description of mesons with one light and one extremely heavy quark when such higher spin mesons are found. The Virasoro conditions select specific $S O(D-1)$ irreps. The asymptotic density of states can be estimated by adapting the HardyRamanujan analysis to a partition of odd integers; the estimate becomes exact as $D$ goes to infinity.
\end{abstract}




\section{Introduction}

Before its reincarnation as a candidate for a fundamental Theory of Everything, String Theory first entered Physics [1], [2] as an attempt to understand the spectrum and interactions of hadrons. This attempt enjoyed considerable success in at least qualitatively accounting for many experimentally observed features of Strong Interactions. For example, the Veneziano amplitude [3] exhibited DolenHorn-Schmid duality [4, appropriate high $s$ and high $t$ behaviors, and linear Regge trajectories. The predictive power of the model was further increased by combining strings with the Quark Model. In this picture, a meson was represented as a quark-antiquark pair connected by a flux tube which behaved like a string. It thus became possible to place observed hadrons in leading or daughter Regge trajectories and predict the masses, spins and internal quantum numbers of mesons that were yet to be observed. In a similar way, Harari-Rosner [5], [6] diagrams provided a useful way of explaining many qualitative properties of particular hadronic processes. This line of research was abandoned upon the almost simultaneous realization that the microscopic theory of Strong Interactions had to be based on an unbroken Yang-Mills theory and that String dynamics were really tailored for 10 or 26 dimensions.

In this note we would like take a modest backward step in history and present a qualitative model for the excited states of mesons consisting of one very heavy quark/antiquark (of the Charmed, Bottom or the Top kind) and one very light antiquark/quark (Up or Down) in terms of a string with one end fixed and one end free. In String Theory language, this system may be approximately described by using Dirichlet boundary conditions (placing the infinitely heavy quark at the origin of the space coordinate system, for example) at one end and Neumann boundary conditions at the other. The resulting system, which we will refer to as a Neumann-Dirichlet, or ND string, is worth examining not only as a guide to some expected properties of mesons with one very heavy quark or antiquark, but also for its novel String theoretic aspects. In fact, our emphasis will be more on the latter question, given that the data on Regge recurrences of such mesons is not available at this stage.

The plan of the paper and some of our main results are as follows. In section 2, we apply the ND conditions on the string in a natural adaptation of the light cone gauge of the NN string to our problem. This leads to what might be called a "rest frame gauge". The ND conditions only allow odd oscillator modes, which are then canonically quantized. The spacetime Hamiltonian and the world-sheet energy momentum tensor are given. In section 3, the Virasoro generators and their algebra for the ND string are worked out. The use of only odd oscillators implies that only even Virasoro mode operators survive and the central term is half that of the usual bosonic string. In section 4, the normal ordering constant for $L_{0}$ is calculated and found to have an opposite sign relative to the usual one; 
consequently, the ND string has no tachyon and no critical spacetime dimension. The absence of even oscillator modes and the tachyon have also been noted earlier in [7]. As excited states are now obtained using odd creation operators only, the spectrum is a subset of that of the NN string. We then present a simple classical model to check the mass-angular momentum relationship for the leading Regge trajectory. The asymptotic density of states can be calculated using a modified version of the Hardy-Ramanujan analysis for the open string. Section 5 ends the paper with concluding remarks.

\section{Quantization}

We start with the Bosonic string action in a $D$-dimensional Minkowski spacetime, hence

$$
S=-\frac{T}{2} \int \mathrm{d} \sigma \mathrm{d} \tau g^{\alpha \beta} \partial_{\alpha} X^{\mu} \partial_{\beta} X^{\nu} \eta_{\mu \nu}
$$

We fix the reparametrization and Weyl invariances of the action in the conformal gauge $g^{\alpha \beta}=\eta^{\alpha \beta}$. The general solutions of the equations of motion

$$
\left(\partial_{\sigma}^{2}-\partial_{\tau}^{2}\right) X^{\mu}=0
$$

can be Fourier expanded as

$$
X^{\mu}=x^{\mu}+l^{2} p^{\mu} \tau+\frac{l}{2} i \sum_{n \neq 0} \frac{\alpha_{n}^{\mu}}{n} \exp -i n(\tau-\sigma)+\frac{l}{2} i \sum_{n \neq 0} \frac{\tilde{\alpha}_{n}^{\mu}}{n} \exp -i n(\tau+\sigma),
$$

where $l$ is a fundamental length introduced to ensure the correct dimensions

for $X^{\mu}$. It is related to the string tension $T$ by $l=\sqrt{\frac{2}{\pi T}}$. The range of the parameter $\sigma$ is taken to be $[0, \pi / 2]$ for reasons which will soon be clear. We locate the infinitely massive quark at the $\sigma=0$ end and also identify this point with the origin of space coordinates through the Dirichlet boundary condition

$$
X^{i}(0, \tau)=0,
$$

which gives $x^{i}=p^{i}=0$ and $\alpha_{n}^{i}=-\tilde{\alpha}_{n}^{i}$ for $i=1,2, . ., D-1$. In modern parlance, we confine one end of the string to a $D 0$-brane $[8]$. We will also take $x^{0}=0$. We adopt the Neumann boundary conditions

$$
\partial_{\sigma} X^{\mu}(\pi / 2, \tau)=0
$$


for the massless end, which require that $\alpha_{n}^{\mu}=0$ for even $n$. This resembles the vanishing of the even parity modes in the familiar ordinary quantum mechanics problem of a particle placed in a "half-oscillator potential", i.e., a well with an infinite barrier at $x=0$ and a harmonic oscillator potential for $x>0$.

Imposing the ND boundary conditions on the space components thus results in

$$
X^{i}=-l \sum_{k} \frac{\alpha_{2 k+1}^{i}}{2 k+1} \exp (-i(2 k+1) \tau) \sin ((2 k+1) \sigma),
$$

with the reality condition yielding $\alpha_{n}^{i \dagger}=\alpha_{-n}^{i}$ as usual.

We now turn to $X_{0}$. Here, an analogy with the light-cone treatment of the $\mathrm{NN}$ string suggests itself. In the light-cone gauge, the $\tau$-parameter is identified with one of the light-cone target coordinates via

$$
X^{+}(\sigma, \tau)=x^{+}+p^{+} \tau,
$$

and this sets the $\alpha_{n}^{+}$operators to zero. Using the constraint equations, one then solves for the $\alpha_{n}^{-}$in terms of the $D-2$ independent transverse operators; hence there is no further need for imposing Virasoro gauge conditions on the states. By contrast, in old covariant quantization, all $X_{\mu}$ are treated on an equal footing, but Virasoro conditions are imposed on the states.

In our case, only one end of the string describes a lightlike worldline, while the other is timelike; hence, the full light-cone gauge treatment is inappropriate. Instead, the natural analog of (6) is to identify the target space and world sheet "times" via

$$
X^{0}=l^{2} p^{0} \tau .
$$

This eliminates the $\alpha_{n}^{0}$ modes and consequently, states with negative norm. We thus adopt an intermediate approach between light-cone and old covariant quantization. There will still be a need to impose constraint equations on the states, although these have been partially pruned by (7). This will lead to a Virasoro algebra with special features which we can already anticipate: By fixing one end of the string in target space, we have broken Poincaré invariance down to $S O(D-1)$ rotational symmetry about the origin of space. Just as in a mechanical problem with an infinite mass, we no longer expect momentum conservation to hold, while energy should still be conserved. This leads us to expect the $L_{1}$ and $L_{-1}$ modes, which involve the momentum operator, will be absent; indeed, since any odd mode can generate these unallowed modes through commutation, only even Virasoro modes should be present. We will see that this actually happens. 
We follow the conventional canonical quantization procedure by requiring that the canonical momenta $\Pi$ and and the coordinates obey the equal time Poisson brackets

$$
\left\{\Pi^{i}(\sigma), X^{j}\left(\sigma^{\prime}\right)\right\}=-\delta^{i j} \delta\left(\sigma-\sigma^{\prime}\right) .
$$

Insertion of (5) into this and the use of the formula

$$
\pi \delta_{2 \pi}\left(\sigma-\sigma^{\prime}\right)=\sum_{k} \exp \left(i(2 k+1)\left(\sigma-\sigma^{\prime}\right)\right)
$$

which may be obtained from the Poisson summation formula (a factor of $\cos \frac{\left(\sigma-\sigma^{\prime}\right)}{2}$ in the denominator of the LHS is omitted since it becomes unity when the argument of the delta function vanishes) yield the expected Poisson brackets ,

$$
\left\{\alpha_{n}^{i}, \alpha_{m}^{j}\right\}=i n \delta_{n,-m} \delta^{i j}
$$

for the Fourier components.

Quantization is effected by replacing Poisson brackets with commutators with the result

$$
\left[\alpha_{n}^{i}, \alpha_{m}^{j}\right]=n \delta_{n,-m} \delta^{i j} .
$$

This is a subalgebra of the bosonic string Heisenberg algebra, $n, m$ being restricted to odd integers.

The Hamiltonian of the theory reads

$$
\begin{aligned}
& H=\frac{T}{2} \int_{0}^{\pi / 2}\left(: \partial_{\tau} X^{\mu} \partial_{\tau} X_{\mu}:+: \partial_{\sigma} X^{\mu} \partial_{\sigma} X_{\mu}:\right) \mathrm{d} \sigma \\
= & \frac{T}{2} \int_{0}^{\pi / 2}\left(: \partial_{\tau} X^{i} \partial_{\tau} X^{i}:+: \partial_{\sigma} X^{i} \partial_{\sigma} X^{i}:-l^{4} p_{0}^{2}\right) \mathrm{d} \sigma
\end{aligned}
$$

with the gauge choice (7). The infinite ground state energy caused by the normal ordering procedure will be calculated and regularized later. The energymomentum tensor

$$
T_{\alpha \beta}=\frac{T}{2}\left(: \partial_{\alpha} X^{\mu} \partial_{\beta} X_{\mu}-\frac{1}{2} \eta_{\alpha \beta} \partial_{\gamma} X^{\mu} \partial^{\gamma} X_{\mu}:\right)
$$

has the components

$$
\begin{aligned}
T_{00} & =T_{11}=\frac{T}{4}\left(: \partial_{\tau} X^{i} \partial_{\tau} X^{i}:+: \partial_{\sigma} X^{i} \partial_{\sigma} X^{i}:-l^{4} p_{0}^{2}\right) \\
T_{01} & =T_{10}=\frac{T}{2}: \partial_{\tau} X^{i} \partial_{\sigma} X^{i}:
\end{aligned}
$$




\section{An even Virasoro algebra}

We now verify our earlier claim that the ND string leads to a Virasoro algebra of even modes only. Imposing open string boundary conditions of the usual NN type identifies the oscillators for the left-moving modes and the right-moving modes, leading to a single set of Virasoro operators. In the ND string, we not only identify left and right-moving oscillators, but in addition exclude the even modes. It is therefore not surprising that half of the Virasoro modes will be eliminated. These have to be the odd ones which cannot close upon commutation, while the even Virasoro modes obviously can. In order to compute them, we first observe that

$$
T_{ \pm \pm}=\frac{1}{2}\left(T_{00} \pm T_{01}\right)=\frac{T}{8}\left(:\left(\partial_{\tau} X^{i} \pm \partial_{\sigma} X^{i}\right)^{2}:-\left(l^{2} p^{0}\right)^{2}\right)
$$

where

$$
\partial_{\tau} X^{i} \pm \partial_{\sigma} X^{i}=\mp l \sum_{m=o d d} \alpha_{m}^{i} \exp (-i n(\tau \pm \sigma))
$$

Thus $T_{++}(\sigma)=T_{--}(-\sigma)$ as in the ordinary open string. Using this property and also the fact that for $\tau=0$

$$
:\left(\partial_{\tau} X^{i} \pm \partial_{\sigma} X^{i}\right)^{2}:=l^{2}: \sum_{n=o d d} \sum_{n=o d d} \exp (-i m \sigma) \alpha_{n}^{i} \alpha_{m-n}^{i}:
$$

where $m$, being the sum of two odd integers, is now necessarily even, we arrive at the even Virasoro mode operators

$$
L_{n}=2 \int_{\frac{-\pi}{2}}^{\frac{\pi}{2}} \exp i n \sigma T_{++} d \sigma .
$$

The constraints $T_{\alpha \beta}=0$ can now be imposed by demanding $L_{n}$ annihilate physical states for $n>0$ and $L_{o}=-\epsilon_{C}$ on the same states. The number $\epsilon_{C}$ will be determined later.

Calling $\alpha_{0}^{\mu}=l p^{\mu}$, we can read off

$$
\begin{aligned}
& L_{0}=-\frac{1}{2}\left(\alpha_{0}^{0}\right)^{2}+\frac{1}{2} \sum_{m=o d d}: \alpha_{m}^{i} \alpha_{-m}^{i}:, \\
& L_{n}=\frac{1}{2} \sum_{m=o d d}: \alpha_{m}^{i} \alpha_{n-m}^{i}: \quad \text { (n even and non-zero) }, \\
& L_{n}=0 \quad(\text { n odd }) .
\end{aligned}
$$

We note that the Virasoro operators have their usual form in terms of the

odd oscillator modes, hence it is clear that they will satisfy the familiar Virasoro 
algebra except perhaps for a change in the central extension term. This term is most easily calculated via the vacuum expectation value

$$
\begin{aligned}
A_{m} & =\left\langle 0\left|\left[L_{m}, L_{-m}\right]\right| 0\right\rangle=\left\langle 0\left|L_{m} L_{-m}\right| 0\right\rangle \\
& =\frac{1}{4}\left\langle 0\left|\sum_{n, k=o d d} \alpha_{m-n}^{i} \alpha_{n, i} \alpha_{-m-k}^{j} \alpha_{k, j}\right| 0\right\rangle \\
& =\frac{1}{4}\left\langle 0\left|\sum_{n, k=o d d} \alpha_{n}^{i} \alpha_{m-n, i} \alpha_{k}^{j} \alpha_{-m-k, j}\right| 0\right\rangle,
\end{aligned}
$$

where $m$ is of course even and positive. The expectation value in the second line vanishes for $k>0$ and for $n>m$, whereas the expectation value in the third line vanishes for $n<0$ and for $k<-m$, yielding

$$
\begin{aligned}
A_{m} & =\frac{1}{4}\left\langle 0\left|\sum_{n=1}^{m-1} \sum_{k=-m+1}^{-1} \alpha_{m-n}^{i} \alpha_{n, i} \alpha_{-m-k}^{j} \alpha_{k, j}\right| 0\right\rangle \\
& =\frac{D-1}{2} \sum_{n=1}^{m-1} n(m-n)
\end{aligned}
$$

where all the summations above are over odd integers. The second line is obtained by successive use of (8) and $D-1=\eta_{i j} \eta^{i j}$ is the dimension of space. The summation in the second line, when taken over all integers, produces the wellknown Virasoro central extension term. Our odd-integer summation, in contrast, yields a modified central extension for the even Virasoro algebra of the form

$$
\left[L_{n}, L_{m}\right]=(n-m) L_{m+n}+\frac{D-1}{24} \delta_{n+m, 0}\left(n^{2}+2\right) n .
$$

It should be noted that the anomaly-free $S L(2, R)$ subalgebra of the open string Virasoro algebra is now reduced to $U(1)$. It is possible to recover a more familiar form for the central term by shifting $L_{0}$ to $L_{0}-\frac{D-1}{16}$. This gives

$$
\left[L_{n}, L_{m}\right]=(n-m) L_{m+n}+\frac{D-1}{24} \delta_{n+m, 0}\left(n^{3}-n\right) .
$$

Note that the coefficient $D$ in the usual Virasoro algebra has been changed to $\frac{D-1}{2}$. The elimination of the odd Virasoro modes is seen to manifest itself as an apparent halving of the number of bosonic coordinates. 


\section{The spectrum}

The on-mass shell condition comes from the zero mode of the Virasoro constraints $T_{\alpha \beta}=0$. Specifically, we derive the masses of the states at the quantum level from

$$
\left(L_{0}+\epsilon_{C}\right)|N, p\rangle=0,
$$

where $\epsilon_{C}$ is the Casimir energy which emerges upon normal ordering of the oscillators. We note that given one end is fixed, all the excitations will have to be analysed in their common rest frame. We thus combine

$$
m^{2}=-p^{\mu} p_{\mu}=-l^{-2} \alpha_{0}^{\mu} \alpha_{0, \mu}=\left(\alpha_{0}^{0}\right)^{2},
$$

with the zero mode Virasoro constraint

$$
\frac{l^{2} m^{2}}{2}=\epsilon_{C}+N=\epsilon_{C}+\sum_{i=1}^{D-1} \sum_{m=1}^{\infty} \alpha_{-(2 m-1)}^{i} \alpha_{2 m-1}^{i} .
$$

In the above, we have performed the normal ordering. The Casimir energy is given by

$$
2 \epsilon_{C}=(D-1) R\left\{\sum_{m=1}^{\infty}(2 m-1)\right\} .
$$

Here $R\{$.$\} denotes the regularized part of the expression inside the brackets.$ We regularize $\epsilon_{C}$ via

$$
2 \epsilon_{C}=(D-1) \lim _{\lambda \rightarrow 0} \sum_{m=1}^{\infty}(2 m-1) \exp (-\lambda(2 m-1)) .
$$

where it is understood that the term that diverges as $\lambda$ goes to zero will be discarded at the end.

Doing the summation, one finds that to second order in $\lambda$

$$
\frac{2}{D-1} \epsilon_{C}=\lim _{\lambda \rightarrow 0} \frac{1}{\lambda^{2}}+\frac{1}{12}+O\left(\lambda^{2}\right),
$$

from which we read off the regularized Casimir energy as

$$
\epsilon_{C}=\frac{D-1}{24}
$$


A quicker way to arrive at the same result is to note that $\zeta(-1)-2 \zeta(-1)=\frac{1}{12}$. The mass formula thus becomes

$$
m^{2}=\frac{1}{l^{2}}\left(\frac{D-1}{12}+2 N\right)
$$

where $N$ is given by

$$
N=\sum_{i=1}^{D-1} \sum_{m=1}^{\infty} \alpha_{-(2 m+1)}^{i} \alpha_{2 m+1}^{i} .
$$

Since $N$ also denotes the highest spin at a given mass, we see that the ND string exhibits linear Regge trajectories, albeit with a Regge slope

$$
\alpha_{N D}^{\prime}=\frac{1}{\pi T}
$$

which is twice as large as the usual $\alpha_{N N}^{\prime}$. The reason behind this can be seen most clearly by comparing an NN string and an ND string both rotating in their rigid, maximum spin modes, the former about its geometrical center, the latter about the fixed end. When data on the excited states of mesons with one light and one extremely heavy quark/antiquark become available, it would be interesting to see whether this slope doubling is experimentally confirmed.

The above conclusions about the Regge trajectories are supported by examining an ND string undergoing rigid rotation in the $X^{1} X^{2}$-plane with

$$
X^{1}=l^{2} p^{0} \sin \sigma \cos \tau, X^{2}=l^{2} p^{0} \sin \sigma \sin \tau, X^{0}=l^{2} p^{0} \tau .
$$

It can readily be verified that these satisfy both the equations of motion and the constraints of vanishing world sheet energy momentum tensor.

The energy of the configuration is

$$
E=M=T \int_{0}^{\frac{\pi}{2}} \partial_{0} X^{0} d \sigma=\frac{l^{2} p^{0} \pi T}{2}
$$

while its angular momentum turns out to be

$$
J=T \int_{0}^{\frac{\pi}{2}}\left(X^{1} \partial_{0} X^{2}-X^{2} \partial_{0} X^{1}\right)=\frac{\pi T\left(p^{0} l^{2}\right)^{2}}{4} .
$$

The rotation being rigid, angular momentum per $M^{2}$ is maximised. The ratio of the former to the latter is the Regge slope, which again comes out to be $\frac{1}{T \pi}$.

We can draw some important conclusions from (30). First of all, the vacuum state with no excitations $(N=0)$, is a positive mass state with $M^{2}=\frac{D-1}{12 l^{2}}$, unlike 
the tachyon in the NN bosonic string. Hence the model has a stable vacuum. The second conclusion concerns the critical dimension for the ND string. From what we have seen so far, it might at first appear that the ND string can exist in any dimension $D>1$. To see why this looks plausible, first recall that we eliminated the negative probability modes at the beginning by identifying $X^{0}$ with $\tau$ and setting all the $\alpha_{n}^{0}$ equal to zero. As the fixed end condition breaks $D$ dimensional Poincaré invariance down to $S O(D-1)$, all we have to check now is whether this reduced rotational symmetry holds in the theory independently of the value of $D$. To show this, we can take over Polchinski's argument [9] used for deriving the critical dimension for the bosonic NN string: By seeking consistency between the Lorentz transformation properties of the states created by the oscillator modes and the mass formula, $D=26$ emerges without having to check the closure of the Lorentz algebra. This amounts to verifying Lorentz covariance in representation space instead of on the operator algebra. In our case, all of the states come in irreducible representations of $S O(D-1)$ since they are created by the $D-1$ space components of the oscillators, and they are all massive, as can be seen from the mass formula. Thus $S O(D-1)$ symmetry is realized on the states regardless of the value of $D$; in fact, as we will see below, the Virasoro conditions select specific $S O(D-1)$ irreps as states. However, the presence of an anomaly term proportional to $D-1$ in our even Virasoro algebra renders this conclusion suspect; we will return to this paradox in the last section.

Turning to the spectrum, the first few examples are as follows:

- $N=1$ :

$$
|1\rangle=\alpha_{-1}^{i}|0\rangle
$$

is a massive vector representation;

- $N=2$ :

$$
|2\rangle=\alpha_{-1}^{i} \alpha_{-1}^{j}|0\rangle
$$

can be decomposed into a symmetric traceless massive rank-2 $S O(D-1)$ tensor and a massive scalar;

- $N=3$ :

$$
|3\rangle=\alpha_{-3}^{i}|0\rangle \oplus \alpha_{-1}^{l} \alpha_{-1}^{j} \alpha_{-1}^{k}|0\rangle
$$

can be decomposed a symmetric traceless rank-3 tensor plus 2 vectors.

At this point one may wonder what role is left for the other Virasoro conditions $L_{n>0}|p h y s\rangle=0$ to play. After all, negative-metric Hilbert space states have already been eliminated at the outset, and linear combinations of the states above can be used to build perfectly physical looking $S O(D-1)$ irreps. The answer, alluded to above, is that not all the possible $S O(D-1)$ irreps at a given $N$ are allowed. The reader can quickly verify that while the $N=1$ state is automatically 
annihilated by $L_{2}, L_{4}, \ldots$, at $N=2$, it is only the rank-2 tensor that satisfies the same Virasoro conditions. Similarly, at $N=3$, the Virasoro conditions select the rank three tensor and the vector created by $\alpha_{-3}^{i}$, while ruling out the vector $\alpha_{-1}^{i} \alpha_{-1}^{k} \alpha_{-1}^{k}$, just as the massive scalar $\alpha_{-1}^{k} \alpha_{-1}^{k}$ at $N=2$ is ruled out. Note that the highest rank tensor state at a given $N$, built out of $N \alpha_{-1}^{i}$ oscillators (with appropriate subtractions of other lower irreps that are created along the way) always exists, and so does the vector state obtained from $\alpha_{-N}^{i}$. Also, one can still obtain states with even $N$ although only combinations of odd-mode creation operators are being used. Thus we can for example write $4=1+1+1+1$ or $4=1+3$, but are not allowed to use $4=4,4=2+1+1,4=2+2$. This means the asymptotic level density as $N \gg 1$ and the Hagedorn temperature of the ND string will not be identical with those of the NN string. We can compute these quantities by slightly modifying the standard techniques given in, say, [10]. One can derive the number of states $d_{n}$ for $N=n$ (or the multiplicity at level $n$ of the partition function $\left.\operatorname{tr} w^{N}\right)$ via

$$
d_{n}=\frac{1}{2 i \pi} \oint \mathrm{d} w w^{-n-1} t r w^{N}
$$

We thus need to compute

$$
\operatorname{tr} w^{N}=g(w)^{-(D-1)}
$$

where $g(w)=\prod_{n=1,3,5, \ldots}\left(1-w^{n}\right)$. There is actually a subtle point here. The original version of this calculation is based on an entirely physical spectrum built out of $D-2=24$ transverse oscillators in the light-cone gauge and therefore the $d_{n}$ represent the true number of physical states at a given $n$. Our calculation, in contrast, will yield the number of all of the states generated by combinations of the $\alpha_{-m}^{i}$ before the Virasoro conditions eliminate the unphysical ones. The relative error in this overestimation however decreases as $1 /(D-1)^{2}$ with increasing $D$ since the unwanted states come from the contraction of the space indices of two oscillators to give a scalar.

Now obviously

$$
g(w)=\frac{f(w)}{f\left(w^{2}\right)}
$$

where $f(w)=\prod_{n=1}^{\infty}\left(1-w^{n}\right)$ is related to the Dedekind eta function by

$$
\eta(\tau)=\exp (i \pi \tau / 12) f(\exp (2 i \pi \tau))
$$

Here $w=\exp (2 i \pi \tau)$ as usual.

Using the S-transformation property of the eta function 


$$
\eta(-1 / \tau)=(-i \tau)^{1 / 2} \eta(\tau)
$$

one gets

$$
g(w)=\frac{1}{\sqrt{2}} w^{1 / 24} q^{1 / 24} \frac{f\left(q^{2}\right)}{f(q)},
$$

where $q=\exp \left(\frac{2 \pi^{2}}{\ln (w)}\right)$.

To investigate the asymptotic density of states we look at the limit $w \rightarrow 1$ for which

or

$$
g(w) \rightarrow A \exp \left(\frac{\pi^{2}}{12 \ln w}\right)
$$

$$
\operatorname{tr} w^{N} \rightarrow A \exp \left(\frac{-\pi^{2}(D-1)}{12 \ln w}\right)
$$

where $A$ is a constant. Using (34) we have

$$
d_{n} \approx \frac{1}{2 i \pi} \oint \mathrm{d} w \exp \left(-\frac{\pi^{2}(D-1)}{12 \ln w}-(n+1) \ln w\right)
$$

Performing the saddle point approximation in (35) around the saddle point

$$
\ln w^{\star}=-\pi \sqrt{(D-1) / 12(n+1)}
$$

one arrives at the asymptotic expression for the level density,

$$
d_{n} \approx \exp \left(\pi \sqrt{\frac{(D-1) n}{3}}\right)(D-1)^{1 / 4} n^{-3 / 4},
$$

where we have only displayed the dependence on the dimension of the space-time and the level $n$, omitting some multiplicative constants. One can compare this with the formula for the NN bosonic string in $D$ dimensions [11]:

$$
d_{n} \approx \exp \left(4 \pi \sqrt{\frac{n(D-2)}{24}}\right) n^{-3 / 4} n^{-(D-2) / 4} .
$$

We observe that going from (36) to (37), the argument of the exponential changes from $\sqrt{n}$ to $\sqrt{2 n}$, This stems from the fact that the ND spectrum is built using only odd spin oscillators, whereas in the NN string both odd and even oscillators contribute to create a state at level $n$. Nevertheless, one can see that 
$d_{n}(N D)>d_{n}(N N)$ for $D=2$ and $D=3$, which is due to the relatively larger contribution of the longitudinal oscillator modes to the ND string for these low dimensions. For higher $D$, the exponential dominates as $n$ goes to infinity and the NN string has the greater multiplicity at a given large $n$.

The level formula (36) shows that the Hagedorn temperature of the ND string is

$$
T_{N D}=\frac{1}{\pi} \sqrt{\frac{3}{2 \alpha_{N N}^{\prime}(D-1)}} .
$$

Here we used the fact that $\alpha_{N D}^{\prime}=2 \alpha_{N N}^{\prime}$. The Hagedorn temperature for the NN string in $D$ dimensions follows from the same formula when $D-1$ is replaced by $D-2$.

\section{Concluding Remarks}

The ND string described above may have some qualitative relevance for describing mesons with one very heavy quark and a very light antiquark. For example, finding such mesons on Regge trajectories with twice the slope of the light meson trajectories would be a confirmation of the picture proposed here. However, we believe the ND string is interesting enough to be considered on its own, just as the original bosonic string proved to have a significance beyond hadron phenomenology. We have shown that it is a bona fide solution of the string equations of motion obeying the constraints and exhibiting a characteristic spectrum: it is essentially the system obtained by viewing the open string from a frame centered at its mid-point and then throwing half of the string away. The constraints lead to a physical Hilbert space via a combination of modified Virasoro conditions and a choice of the $\tau$ parameter which eliminates negative probabilities. However, there are three questions that need to be answered; the first two turn out to be related. The first is the unaddressed expectation that the problem should involve an infinite mass associated with the fixed end. The second and the third involve the apparent conflict between two facts: On the one hand, the theory appears to be unitary and $S O(D-1)$ invariant for any $D$, while on the other hand, the presence of a non-zero anomaly term in the Virasoro algebra casts doubt on the possibility of imposing the constraints $T_{\alpha \beta}=0$ in a consistent way independently of the value of $D$. The answer to the first two questions is that although the ND string, unlike the bosonic or supersymmetric strings, does not sharply require a specific dimension such as 26 or 10, it nevertheless prefers $D$ to be as high as possible.

In relation to the first question, we note that the mass of the scalar ground state is proportional to $D-1$; hence an infinite $D$ is consistent with one end of 
the string being immovable. Another consequence of this infinite $D$ limit is that the group $S O(D-1)$ with $(D-1)(D-2) / 2$ generators and the full inhomogenous Lorentz group with only $2 D-1$ additional generators "merge up to order $1 / D$ " ; thus in a sense, the ND string recovers its Poincaré invariance as $D$ goes to infinity. This is evidenced also by a fact we observed earlier: the overestimation in the density of states vanishes like $1 / D$. Interestingly, In the rather different approach to the problem taken in [7], $D$ has to be taken to infinity to make a saddle point calculation possible.

The third question, namely the presence of the anomaly term in the Virasoro algebra, gets affected by the above choice of $D$; the coefficient of the anomaly is now infinite. Since conformal invariance is violated by the immobile end, a conformal anomaly has to appear somewhere in the model; its becoming infinite is perhaps an indication is that like most other infinite quantities in physics, it is to be disregarded! Indeed, the problems normally associated with the conformal anomaly have already been solved: there are no negative norm or zero norm states, and the Virasoro conditions have organized and selected states into $S O(D-1)$ irreps.

Finally, there is the intriguing question, which we intend to investigate, of whether this system of half a bosonic string has in some sense a fermionic character. This is suggested not only by the general fact that a pair fermions behaves like a boson, but also by some specific hints. Instead of restricting the range of $\sigma$ to $\frac{\pi}{2}$ and working with odd oscillator modes, one could keep the range as $\pi$ and work with half-odd integer Neveu-Schwarz-like modes; recall also the halving of the number of dimensions appearing in front of the Virasoro anomaly noted earlier.

Note added: After submitting the above note to the Web, we were notified of two earlier papers [12], [13] involving ideas related to ours. Ref. 112] is concerned with off-shell states in Dual Resonance models; in particular, it employs an $R^{\mu}$ field with Neveu-Schwarz-like half integer but commuting modes for the factorization of amplitudes with one off-shell particle. Had we chosen the interval $[o, \pi]$ instead of $\left[o, \frac{\pi}{2}\right]$ for our $X^{\mu}$ 's, we would have also obtained half-integer commuting modes instead of our odd-integer ones. In [13], the $D$ dimensions of spacetime are separated into $D_{o}$ ordinary and $D_{E}$ extraordinary components for the purpose of changing the intercept and the dimension to physical values. The analysis is based on maintaining Poincaré covariance in the ordinary dimensions while breaking it in the others by fixing one end of the extraordinary $X^{\mu}$ coordinates. In contrast, Poincaré covariance is entirely reduced to $S O(D-1)$ covariance in our work.

Acknowledgements We are grateful to D. Fairlie and W. Siegel for bringing their papers to our attention. We are grateful to S. Arapoğlu, M. Arık, C. Deliduman, R. Güven and T. Turgut for useful discussions. 


\section{References}

[1] Y. Nambu, "Quark model and the factorization of the Veneziano amplitude", in Symmetries and Quark Models, ed. R. Chand, Gordon and Breach, p. 269 (1970); Y. Nambu, "Duality and hadrodynamics", Lectures at the Copenhagen Symposium

[2] L. Susskind, Phys. Rev.D1 (1970)1182

[3] G. Veneziano, Nouvo Cim. 57A(1968)190

[4] R. Dolen, D. Horn and C. Schmid, Phys. Rev. 166 (1968)1768

[5] H. Harari, Phys. Rev. Lett. 22 (1969)562

[6] J. Rosner, Phys. Rev. Lett. 22 (1969)689

[7] H. Kleinert, G. Lambiase and V.V. Nesterenko, Phys.LettB 384, 313-317, (1996)

[8] J. Polchinski, String Theory, Volume 1, p. 261, Cambridge University Press (1998)

[9] J. Polchinski, String Theory, Volume 1, p. 21, Cambridge University Press (1998)

[10] M.B. Green, J.H. Schwartz, E. Witten, Superstring Theory Vol.1., p. 116, Cambridge University Press, (1987)

[11] M.B. Green, J.H. Schwartz, E. Witten, Superstring Theory Vol.1., p.118, Cambridge University Press, (1987)

[12] E. Corrigan and D. B. Fairlie, Nucl.Phys. B91 (1975)527

[13] W. Siegel, Nucl.Phys. B109 (1976)244 